\title{
Study on a noninvasive method for rapid screening Human Serum albumin injectables by Raman spectroscopy
}

\author{
Yu Zhao*, Nan Ji ${ }^{\dagger}$ Xiuji Li*, Jifeng Hou*, Min Li*, Yongjian Yang*, \\ Ping Tian ${ }^{\dagger}$, Xinmei Sun ${ }^{\dagger}$, Xinyue Xiao* and Lihui Yin ${ }^{*}$, \\ *National Institutes of Food and Drug Control (NIFDC) \\ No. 2 Tiantan Xi Li, Beijing, P. R. China \\ ${ }^{\dagger}$ Sichuan Vspec Technologies Co. Ltd. Beijing Office \\ No. 2 BeiTaiPingZhuang Road, Beijing, P. R. China \\ \$Shanghai Institute for Food and Drug Control (SIFDC) \\ No. 1500 Zhangheng Road, Shanghai, P. R. China \\ \$yinlihui@vip.163.com
}

Received 22 November 2015

Accepted 18 January 2016

Published 10 March 2016

\begin{abstract}
Human serum albumin (HSA) injectable product is a severely afflicted area on drug safety due to its high price and restricted supply. Raman spectroscopy performances high specificity on HSA detection and it is even possible to determine HSA injectable products noninvasively. In this study, we developed a noninvasive rapid screening method for of HSA injectable products by using portable Raman spectrometer. Qualitative models were established by using principal component analysis combined with classical least squares (PCA-CLS) algorithm, while quantitative model was established by using partial least squares (PLS) algorithm. Model transfer in different instruments of both the same and different apparatus modules was further discussed in this paper. A total of $34 \mathrm{HSA}$ injectable samples collected from markets were used for verification. The identification results showed $100 \%$ accuracy and the predicted concentrations of those identified as true HSA were consistent with their labeled concentrations. The quantitative results also indicated that model transfer was excellent in the same apparatus modules of Raman spectrometer at all concentration levels, and still good enough in the different apparatus modules although the relative standard deviation (RSD) value showed a little increasing trend at low HSA concentration level. In conclusion, the method was proved to be feasible and efficient for screening HSA injections, especially on its screening speed and the consideration of glass containers. Moreover, with inspiring results on the model transfer, the method could be used as a universal screening mean to different Raman instruments.
\end{abstract}

$\S_{\text {Corresponding author. }}$

This is an Open Access article published by World Scientific Publishing Company. It is distributed under the terms of the Creative Commons Attribution 4.0 (CC-BY) License. Further distribution of this work is permitted, provided the original work is properly cited. 
Keywords: Human serum albumin; Raman noninvasive rapid screening method; principal component analysis combined with classical least squares; partial least squares; model transfer.

\section{Introduction}

- Human serum albumin (HSA) has acted as a pharmaceutical preparation for clinical use for more than 50 years. HSA injectables are protein preparations separated from the plasma of healthy humans. They are used to treat some extremely serious diseases in clinic, such as secondary shock and encephaledema caused by hemorrhage, burn or trauma, hydrops and ascites caused by liver cirrhosis or nephrosis, increased intracranial pressure caused by brain injury, hyperbilirubinemia of newborn, hypoproteinemia and so on. ${ }^{1}$ Because the demand is great, but the supply is restricted, the price of HSA injectables is becoming extremely high, which motivated counterfeit criminals by large anticipated profits. In this case, a large number of fake and counterfeit incidents had been found in clinic and medicine trading market. ${ }^{2}$ That has been endangering the public life safety. Therefore, HSA product is a severely afflicted area on drug safety and a more specific rapid screening method is badly in need especially on-site use.

- At present, there are many measurement methods for HSA determination, ${ }^{1,3-8}$ such as colorimetry, polarimetry, Kjeldah, ultraviolet (UV) spectroscopy, liquid chromatography (LC), liquid chromatography-mass spectrometry (LC-MS), capillary electrophoresis (CE) and Raman spectroscopy. Amongst these methods, the first four were broadly used, especially the molybdate colorimetry method, ${ }^{1}$ which is the most widely-used rapid screening method for HSA detection. However, these methods do not have enough specificity for HSA. On the other hand, although the LC, LC-MS and CE methods are specific enough for HSA, the complicated operation and consumption of solvents limit these methods for field use. Raman method has both benefits of the highly specific on protein and easy-to-use, and is considered as an efficient means for determination of HSA. ${ }^{7,8}$ Moreover, with the development of hardware technique, Raman instruments are becoming more miniature and portable, which makes the application of Raman spectroscopy a bright and broad future on the field test. In our present studies, we developed a noninvasive Raman method for detecting the liquid injectables without taking out the glass containers. ${ }^{9-11}$ However, these works were performed in the chemical drug injectables, whose active pharmaceutical ingredient (API) signals were strong enough for isolating and extracting data process. So it was a serious challenge to transplant that method to HSA injectable products.

- In order to realize rapid screening of HSA injectable products in a noninvasive way, we developed a convenient and effective method for portable Raman spectrometer. In this paper, two main problems were solved. One was whether the signal from HSA could be extracted from the mixed spectra and distinguished from other substances. The other was whether the model established could be directly applied on different end-users' instruments.

\section{Materials and Methods}

\subsection{Samples}

HSA standard substance was acquired from SigmaAldrich with the purity of $96 \%$. Analytical purity reagents twain 80 , pure water, sodium chloride injection and compound amino acid injection (18AA) were used as the negative reference. And human immunoglobulin (HI) injections and egg white were used as negative challenge protein samples. Both real and counterfeit HSA injections collected from markets were used for the verification.

\subsection{Solution preparation}

Twain 80 and egg white were prepared into water solution for negative challenges use. Three series HSA solutions were prepared for the quantitative model. The first series concentrations of $2.0 \%, 4.9 \%$, $6.5 \%, 9.8 \%, 11.7 \%, 14.6 \%, 19.5 \%$ and $23.8 \%(\mathrm{w} / \mathrm{v})$ were made by dissolving HSA standard substance with water. The second series concentrations of $5 \%$, $9 \%, 13 \%, 17 \%, 21 \%, 23 \%$ and $25 \%$ (w/v) were made 
by mixing two known concentration HSA injectables of $5 \%(\mathrm{w} / \mathrm{v})$ and $25 \%(\mathrm{w} / \mathrm{v})$, respectively. The last series concentrations of $4.8 \%, 5.8 \%, 7.1 \%, 8.7 \%$, $10.7 \%, 13.1 \%, 16.0 \%$ and $19.5 \%(\mathrm{w} / \mathrm{v})$ were made by diluting a known concentration HSA injectable sample of $19.5 \%(\mathrm{w} / \mathrm{v})$. Each solution was contained in ampoules, vials and bottles respectively to simulate the actual determination process.

\subsection{Apparatus and software}

Totally five sets of portable Raman spectrometers, No. 197121 (Metage OPAL-2800, Metage Scientific, UK), Nos. 685001, 685002, 685004 (OPAL-3000, Metage Scientific, UK) and No. 140603308 (iRaman-plus, BWTEK, USA) were used for this study. All spectrometers were equipped with fiber optic probes and $785 \mathrm{~nm}$ diode laser excitation sources. A specially designed sample compartment was utilized to assure measurement under dark environment and samples in ampoule, vials or bottle were at the focus point of the probe. RFDI software (version 2.0, Sichuan Vspec Technologies Co. Ltd.) was used for both data collection and model building. First derivative pre-processing was applied during method development.

\subsection{Raman spectra collections}

Offset correction, $x$-axis correction and $y$-axis correction were done on each Raman spectrometer before collecting the spectra. Raman spectra were collected with the output power of $400 \mathrm{~mW}$, a resolution of $4.5 \mathrm{~cm}^{-1}$ and total scan time of $100 \mathrm{~s}$ in the spectral range of $200-3000 \mathrm{~cm}^{-1}$. HSA samples in their original containers (ampoules, vials or bottles) were positioned in the specially designed sample compartment and measured directly through the glass containers. The API reference standard water solutions prepared in ampoules, vials and bottles were measured as same as the samples. Water spectra were collected in $1 \mathrm{~cm}$ quartz cuvettes.

\subsection{Data analysis}

In order to monitor and control the differences between separate Raman instruments, standardization had been done based on the offset, $x$-axis and $y$-axis corrections. Four performance indexes wavelength accuracy and precision, signal to noise and relative intensity - were utilized to quantify the differences. Thus, the differences between instruments could be controlled to a relatively acceptable level by means of setting thresholds of the four indexes. The thresholds are adjusted according to experiences and experimental feedbacks. ${ }^{12}$

Principal component analysis combined with classical least squares (PCA-CLS) algorithm was utilized to fit the pure HSA signal extracting from the injectable mixed signals with glass packages for the noninvasive determination of HSA injections. The signals from the glass containers contributed over $90 \%$ to the noninvasive spectra and varied from different manufactures. In order to achieve the best results of fitting, PCA was done on a wide range of glass spectra before the CLS was performed. ${ }^{9-11}$ With the extracted HSA spectra, we could build the models for both identification and quantification. The identification was done by means of calculating correlation coefficients between the HSA spectra extracted from samples and the reference spectrum collected from the HSA standard solution. The nearer the correlation coefficient was to one, the more the HSA signals extracted from the injectable sample were similar to the reference. According to the market investigation, four kinds of substances - twain 80, pure water, sodium chloride injection and compound amino acid injection (18AA) - were chosen as the negative reference for both threshold setting and validation. A "positive or negative" identification result could be achieved by using the thresholds, where positive is for a HSA product and negative is not.

The quantification was performance based on the results of the identification. If the identification results of the injectables were positive, further work would be performed in the calculation of the concentration of the HSA. And the partial least squares (PLS) model for quantification was built by using the known concentration HSA samples prepared. The leave-one-out cross-validation was used to select optimal parameters and avoid over fitting. To achieve better fitting results and calculation accuracy, pretreatment method of vector normalization was utilized on the Raman spectra to eliminate the differences coming from the different measurement conditions on base of controlling the quantitative performance indexes of different instruments. The optimal parameters were evaluated by considering the coefficient of determination for calibration 
$\left(\mathrm{R}^{2}\right)$ and root mean square error of calibration (RMSEC).

Finally, the HSA products collected from the market were used for the validation of the methods we built.

\section{Results and Discussion}

\subsection{Signal extraction for identification}

As mentioned in previous study, ${ }^{9-11}$ the signals from the glass containers contribute most in noninvasive Raman spectra. In the HSA injectable products, there are primarily three kinds of glass containers, ampoule, vial and bottle. In fact, the walls of big bottles are much thicker than that of ampoules, vials and small bottles, so the signals of HSA are relatively weaker when contained in these bottles. As shown in Fig. 1, the Raman spectra before extraction of the pure HSA water solution should have four peaks of $1654 \mathrm{~cm}^{-1}, 1449 \mathrm{~cm}^{-1}, 1341 \mathrm{~cm}^{-1}$ and $1003 \mathrm{~cm}^{-1}$, consistent with that contained in ampoule with thin wall, but that of HSA samples contained in vial and big bottle with thick walls showed up only three peaks and one peak, respectively. It is obvious that HSA signals will be easier to be extracted when the products contained in ampoules than in vials and bottles. Generally, we consider the wall-thickness from 1 to $3 \mathrm{~mm}$ as "thick wall". On the other hand, the materials of glass are varied, which have strong Raman signals that could also influent the extraction for identification. Therefore, in order to achieve better results for the actual samples, we established two HSA injectable identification models, which could be chosen according to the thickness of containers (as shown in Table 1).

\section{Verification of Identification Models}

The investigation on the HSA in markets indicated that the fake HSA injectables were mainly twain 80, amino acids, sodium chloride injection and water for injection. In these counterfeit cases, twain 80 were the most similar on apparent characteristic and compound amino acids were the most similar on molecular structures. However, the Raman spectra of above substances had significant differences with that of HSA. Figure 2 showed the spectra of twain 80 water solution and compound amino acid injection comparing with HSA water solution. It could be seen from Fig. 2 that bands around $1656 \mathrm{~cm}^{-1}$ (amide band I), $1448 \mathrm{~cm}^{-1}$ (amide band II), $1346 \mathrm{~cm}^{-1}$ (amide band III) and peak $1003 \mathrm{~cm}^{-1}$ (phenyl in side chain) were considered as the

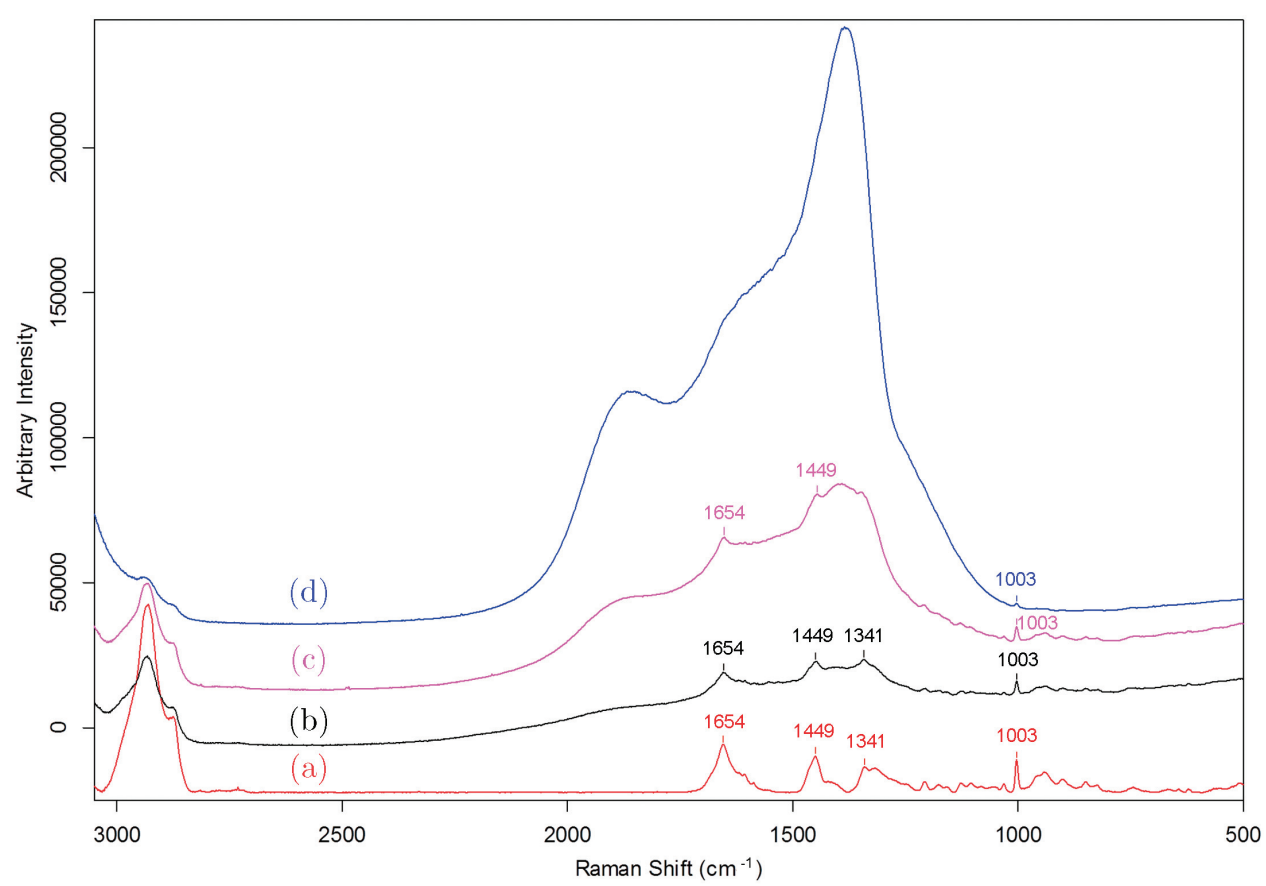

Fig. 1. The Raman spectra before extraction, where (a) is of the pure HSA water solution contained in quartz cuvettes, (b) is of the sample contained in ampoule, (c) is of the sample contained in vial and (d) is of the sample contained in big bottle with thick wall. 
Table 1. Identification models and relative parameters according to the thickness of containers' wall.

\begin{tabular}{lcccc}
\hline Models & Thickness of containers' wall & $\begin{array}{c}\text { Calibration } \\
\text { range }\left(\mathrm{cm}^{-1}\right)\end{array}$ & Spectra pretreatment & $\begin{array}{c}\text { Thresholds for } \\
\text { identification }\end{array}$ \\
\hline ID model 1 & $\begin{array}{c}\text { Thin (ampoules, vial or some } \\
\text { small bottles) }\end{array}$ & $1800-600$ & Baseline correction & 0.95 \\
ID model 2 & Thick (most bottles) & $1050-980$ & Baseline correction & 0.9 \\
\hline
\end{tabular}

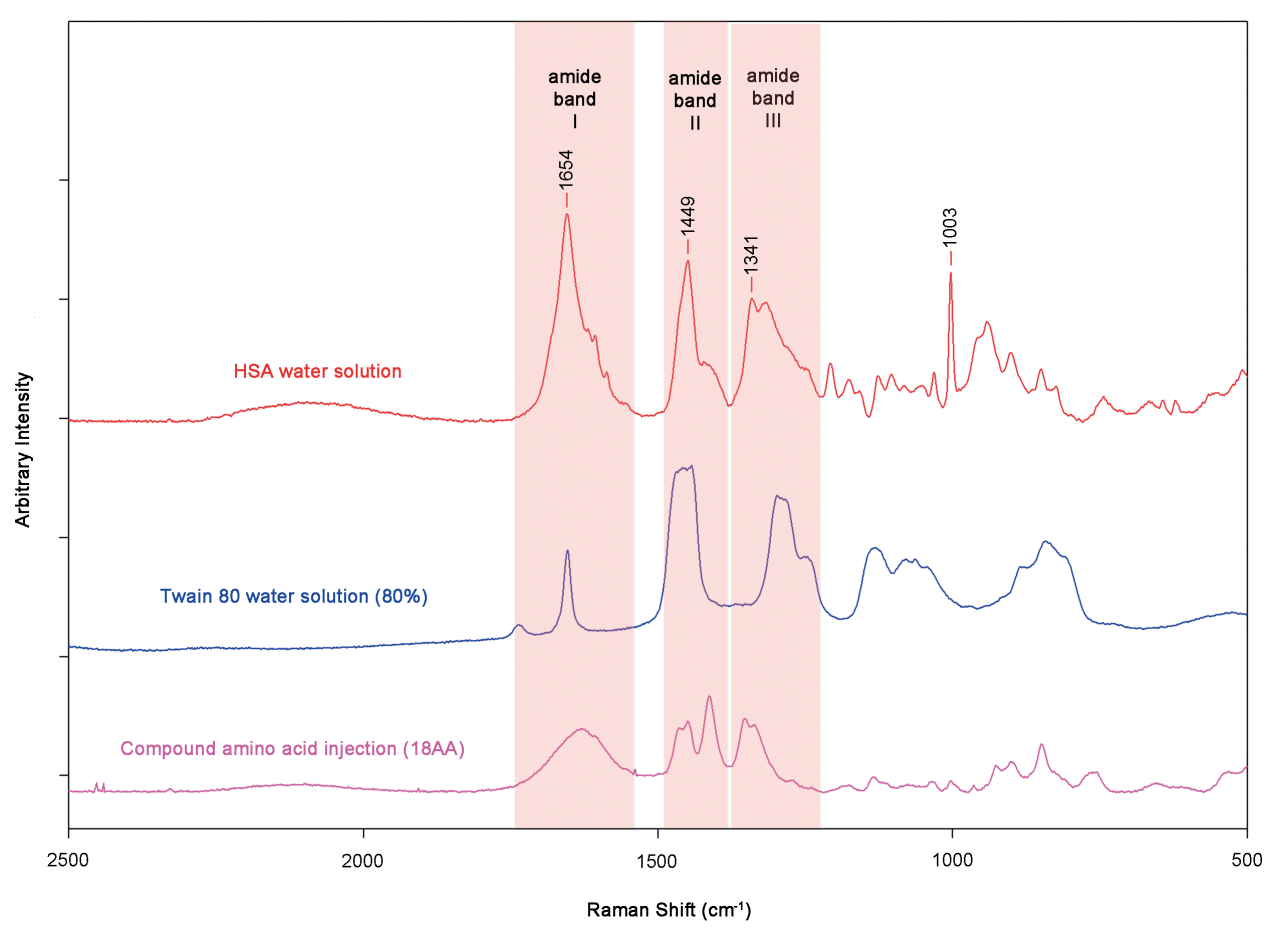

Fig. 2. Characteristic bands and peak of HSA spectrum comparing with spectra of twain 80 water solution and compound amino acid injection (18AA).

characteristic bands of HSA to distinguish from other small molecular substances. Meantime, when we used these small molecular substances to verify our identification models, the results showed us that it is easy to distinguish them from the HSA according to the correlation coefficients. Those with correlation coefficients lower than 0.9 were considered as negative results while others with correlation coefficients higher than 0.9 or even 0.95 were considered as positive results. Table 2 showed the correlation coefficients output from the identification model for both negative challenges and positive testing results.

Despite the fact that present investigations have not found cases of using other protein to counterfeit HSA, a foreseeable negative challenges had been done on the models in this study by using HI and egg white. As Fig. 3 shown, bands around
$1656 \mathrm{~cm}^{-1}$ (amide band I), $1448 \mathrm{~cm}^{-1}$ (amide band II), $1346 \mathrm{~cm}^{-1}$ (amide band III) and peak $1003 \mathrm{~cm}^{-1}$ (phenyl in side chain) were also considered as the characteristic bands of HSA to distinguish from another proteins. When verifying the models, the correlation coefficients of $\mathrm{HI}$ and egg white were lower than 0.9. Thus, both the spectra and models told significant differences between HSA and other protein molecules. Table 2 showed both the negative challenge and positive testing results of the potential counterfeit substances above.

Moreover, 32 known HSA injection samples collected from markets were additionally used for verification of the identification models. All these samples were identified by LC-MS method and had testing reports as reference. Two more HSA injectable samples marked "Real" but without testing reports were also determined. The models were 
Table 2. Negative challenges and positive testing results on potential counterfeit substances.

\begin{tabular}{lccc}
\hline Substances & Mean correlation coefficient & Repetition testing & Identification results \\
\hline Twain 80 & 0.2783 & 6 & negative \\
Single amino acid injection & 0.5029 & 6 & negative \\
Compound amino acid injection & 0.4547 & 6 & negative \\
Water for injection & -0.1754 & 6 & negative \\
Sodium Chloride Injection & -0.2148 & 6 & negative \\
Egg white & 0.7836 & 6 & negative \\
HI injection & 0.8523 & 6 & negative \\
HSA injection & 0.9854 & 6 & positive \\
\hline
\end{tabular}

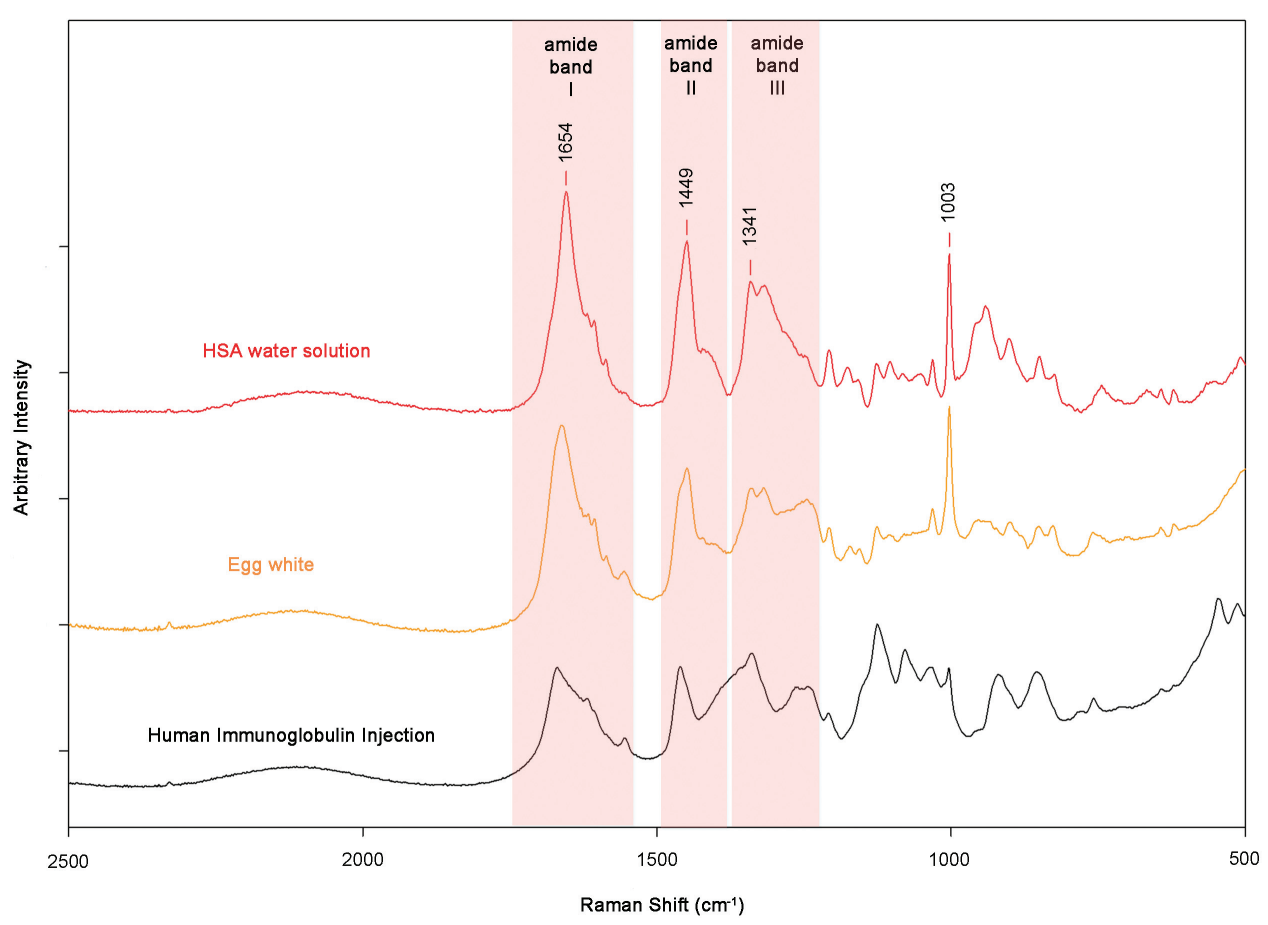

Fig. 3. Characteristic bands and peak of HSA spectrum comparing with spectra of other protein molecules (HI water solution and egg white).

chosen according to the thickness of glass containers. Final positive or negative results were evaluated by comparing the correlation coefficients with thresholds of each model. As shown in Table 3, all the identification results were consistent with the reference.

\section{Quantification and Model Transfer}

The quantitative model was performed on the prepared HSA water solutions with known concentrations. With the pretreatment of vector normalization and spectra ranges of 3200$2500 \mathrm{~cm}^{-1}$ and $1146-800 \mathrm{~cm}^{-1}$, the PLS model produced an $\mathrm{R}^{2}$ of 0.9911 in calibration. As shown in Fig. 4, the cross-validation results performed well in linear with the corresponding minimum RMSECV value of 0.64 .

Quantitative analysis should be based on the results of identification. Only samples that were identified as positive would go further for quantification. Samples No. 1-5 in Table 2 were used for the verification of the PLS model (Table 4). The predicted concentrations were on the same level of the labeled concentrations. As a screening method, requirements on quantification are more flexible compared with other analytical methods. In our screening method, only predicted concentration and labeled concentrations were provided as final results, without giving a conclusion of fake or 
Table 3. Identification results of real and fake HSA injectatble products collected from the market.

\begin{tabular}{|c|c|c|c|c|c|c|c|c|}
\hline No. & $\begin{array}{c}\text { Labeled } \\
\text { manufactures }\end{array}$ & $\begin{array}{l}\text { Sample } \\
\text { source }\end{array}$ & Batch no. & $\begin{array}{l}\text { Labeled } \\
\text { amount }\end{array}$ & $\begin{array}{c}\text { Known } \\
\text { Real/Fake }\end{array}$ & Chosen models & $\begin{array}{c}\text { Correlation } \\
\text { coefficient }\end{array}$ & $\begin{array}{c}\text { Identification } \\
\text { results }\end{array}$ \\
\hline 1 & $\mathrm{~A}$ & \multirow[t]{5}{*}{ Shanghai } & 20060924 & $20 \%$ & Real & ID model 1 & 0.9718 & positive \\
\hline 2 & $\mathrm{~A}$ & & 20060929 & $20 \%$ & Fake & ID model 1 & 0.3235 & negative \\
\hline 3 & $\mathrm{~B}$ & & 20120835 & $20 \%$ & Real & ID model 1 & 0.9650 & positive \\
\hline 4 & $\mathrm{C}$ & & 200804030 & $10 \%$ & Real & ID model 1 & 0.9771 & positive \\
\hline 5 & $\mathrm{D}$ & & VNA1J125 & $5 \%$ & Real & ID model 2 & 0.9634 & positive \\
\hline 6 & $\mathrm{E}$ & \multirow[t]{4}{*}{ Nanning } & 201104113 & $20 \%$ & Fake & ID model 2 & 0.2154 & negative \\
\hline 7 & $\mathrm{E}$ & & 201203115 & $20 \%$ & Fake & ID model 2 & 0.2567 & negative \\
\hline 8 & $\mathrm{~F}$ & & 201205115 & $20 \%$ & Fake & ID model 2 & 0.2291 & negative \\
\hline 9 & $\mathrm{~F}$ & & 201302077 & $20 \%$ & Fake & ID model 2 & 0.2618 & negative \\
\hline 10 & G & \multirow[t]{23}{*}{ Jilin } & 20060434 & $20 \%$ & Fake & ID model 1 & 0.3247 & negative \\
\hline 11 & $\mathrm{G}$ & & 20061144 & $20 \%$ & Fake & ID model 1 & 0.2844 & negative \\
\hline 12 & $\mathrm{H}$ & & 43644411A & $20 \%$ & Fake & ID model 1 & 0.1903 & negative \\
\hline 13 & I & & $200606 \mathrm{~A} 026$ & $20 \%$ & Fake & ID model 2 & 0.2220 & negative \\
\hline 14 & $\mathrm{~J}$ & & 20060308 & $20 \%$ & Fake & ID model 1 & 0.4766 & negative \\
\hline 15 & $\mathrm{I}$ & & 200601003 & $20 \%$ & Fake & ID model 2 & 0.3389 & negative \\
\hline 16 & $\mathrm{~K}$ & & 200609002 & $20 \%$ & Fake & ID model 1 & 0.2811 & negative \\
\hline 17 & $\mathrm{~J}$ & & 20050924 & $20 \%$ & Fake & ID model 1 & 0.2994 & negative \\
\hline 18 & $\mathrm{~J}$ & & 20060308 & $20 \%$ & Fake & ID model 1 & 0.4626 & negative \\
\hline 19 & $\mathrm{G}$ & & 20060434 & $20 \%$ & Fake & ID model 1 & 0.3829 & negative \\
\hline 20 & $\mathrm{C}$ & & 200602016 & $20 \%$ & Fake & ID model 1 & 0.3465 & negative \\
\hline 21 & $\mathrm{~L}$ & & 200604019 & $20 \%$ & Fake & ID model 1 & 0.4363 & negative \\
\hline 22 & $\mathrm{~K}$ & & 200609002 & $20 \%$ & Fake & ID model 1 & 0.2846 & negative \\
\hline 23 & $\mathrm{~L}$ & & 200609042 & $20 \%$ & Fake & ID model 1 & 0.2848 & negative \\
\hline 24 & $\mathrm{I}$ & & $20050904 \mathrm{~A} 0$ & $20 \%$ & Fake & ID model 2 & 0.2574 & negative \\
\hline 25 & I & & $20050905 \mathrm{~A} 0$ & $20 \%$ & Fake & ID model 2 & 0.1575 & negative \\
\hline 26 & I & & $200512 \mathrm{~A} 063$ & $20 \%$ & Fake & ID model 2 & 0.3664 & negative \\
\hline 27 & $\mathrm{G}$ & & 20061144 & $20 \%$ & Fake & ID model 1 & 0.2687 & negative \\
\hline 28 & $\mathrm{I}$ & & 200604A018 & $20 \%$ & Fake & ID model 2 & 0.4621 & negative \\
\hline 29 & I & & 200607A031 & $20 \%$ & Fake & ID model 2 & 0.2684 & negative \\
\hline 30 & $\mathrm{H}$ & & $32644411 \mathrm{~A}$ & $20 \%$ & Fake & ID model 1 & 0.5824 & negative \\
\hline 31 & $\mathrm{H}$ & & $436644411 \mathrm{~A}$ & $20 \%$ & Fake & ID model 1 & 0.4168 & negative \\
\hline 32 & $\mathrm{~A}$ & & 20060924 & $20 \%$ & Fake & ID model 1 & 0.2586 & negative \\
\hline 33 & $\mathrm{~A}$ & \multirow[t]{2}{*}{ NIFDC } & 20060929 & $20 \%$ & marked "Real" & ID model 1 & 0.9537 & positive \\
\hline 34 & $\mathrm{D}$ & & A250A6661 & $20 \%$ & marked "Real" & ID model 2 & 0.9621 & positive \\
\hline
\end{tabular}

counterfeit. That is because the practical situation will be more complex than figures themselves.

Furthermore, a rapid screening method needs to be adaptable to a wide and varied range. So the models, we established should have to be transferred in different instruments instead of re-building the model each time measurement conditions changed. In this study, model transfer was researched in both the same apparatus modules and different apparatus modules. Instrument No. 685001 (Opal3000) was the one that the quantitative model being established on, and instruments No. 685002 and 685004 were utilized to validate the model transfer in different instruments of the same apparatus modules, while instruments No. 140603308
(iRaman-plus) and No. 197121 (Opal2800) were utilized to validate the model transfer in portable Raman spectrometers of different apparatus modules. As indicated in Table 4, the RSD values, on behalf of the model transfer results in the apparatus modules of Opal3000, were less than $1 \%$ at relatively high HSA concentration levels, while was $2.25 \%$ at a lower concentration level of $5 \%$. As for different apparatus modules, the RSD value was $2 \%$ in higher concentration level and $6.17 \%$ at lower concentration level. These results indicated that model transfer was excellent in the same apparatus modules of Raman spectrometer at all concentration levels, while still good enough in the different apparatus modules although the RSD values 


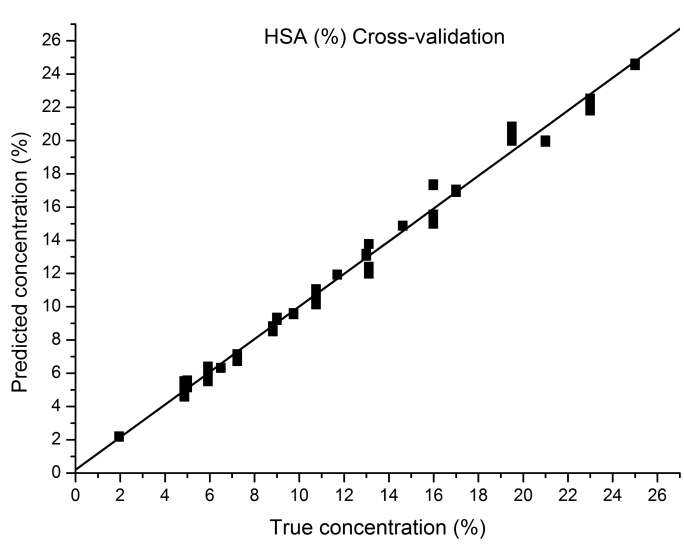

(a)

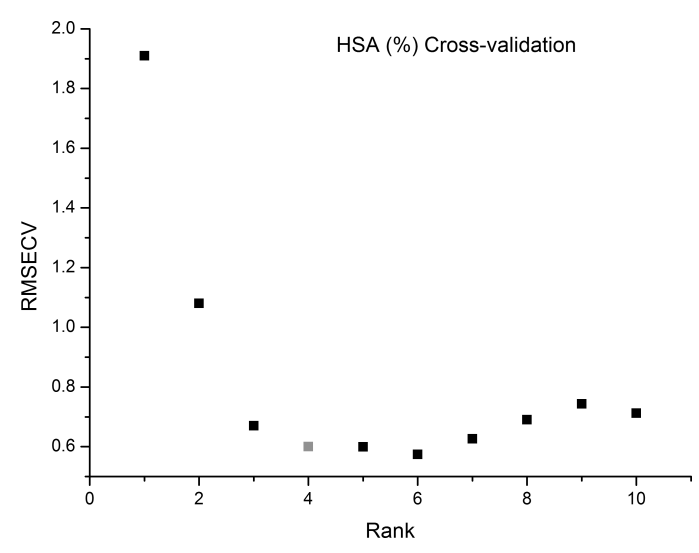

(b)

Fig. 4. Cross-validation results of the PLS model: (a) predicted concentrations vs actual concentrations; (b) minimum RMSWCV is 0.64 in the fourth rank.

showed a little increasing trend at low HSA concentration levels. This phenomenon is for the reason that sometimes signal-to-noise of spectra on low concentration level becomes worse due to the dataprocessing failure on glass containers, which will lead to a negative influence on the performance of quantification model. On this occasion, if this noninvasive way did not work on HSA concentration lower than $5 \%$, the way of taking HSA samples out of the glass containers could also be a wise choice. Moreover, as samples No. 33 and 34 were considered to be positive by the identification models, their concentrations were also predicted by the quantification model (as shown in Table 4) and the predicted concentrations were correspondent to labeled information as well.

Table 4. Verification results of quantification and model transfer.

\begin{tabular}{|c|c|c|c|c|c|c|}
\hline Sample no. & $\begin{array}{l}\text { Identification } \\
\text { results }\end{array}$ & $\begin{array}{c}\text { Labeled } \\
\text { concentration } / \%\end{array}$ & $\begin{array}{c}\text { Predicted } \\
\text { concentration } / \%\end{array}$ & $\begin{array}{c}\text { Raman } \\
\text { instrument } \\
\text { used }\end{array}$ & $\begin{array}{c}\text { Model transfer in the } \\
\text { same apparatus } \\
\text { model RSD } / \%\end{array}$ & $\begin{array}{l}\text { Model transfer } \\
\text { in different } \\
\text { apparatus } \\
\text { models RSD } / \%\end{array}$ \\
\hline \multirow[t]{5}{*}{1} & \multirow[t]{5}{*}{ positive } & \multirow[t]{5}{*}{20} & 16.94 & 197121 & \multirow[t]{2}{*}{ - } & \multirow{5}{*}{1.24} \\
\hline & & & 16.64 & 685001 & & \\
\hline & & & 16.56 & 685002 & \multirow[t]{2}{*}{0.24} & \\
\hline & & & 16.60 & 685004 & & \\
\hline & & & 16.37 & 140603308 & 一 & \\
\hline \multirow[t]{5}{*}{2} & \multirow[t]{5}{*}{ positive } & \multirow[t]{5}{*}{20} & 19.46 & 197121 & \multirow[t]{2}{*}{ - } & \multirow{5}{*}{0.47} \\
\hline & & & 19.54 & 685001 & & \\
\hline & & & 19.67 & 685002 & \multirow[t]{3}{*}{0.28} & \\
\hline & & & 19.58 & 685004 & & \\
\hline & & & 19.68 & 140603308 & & \\
\hline \multirow[t]{5}{*}{4} & \multirow[t]{5}{*}{ positive } & \multirow[t]{5}{*}{10} & 10.29 & 197121 & \multirow[t]{2}{*}{ - } & \multirow{5}{*}{1.13} \\
\hline & & & 10.32 & 685001 & & \\
\hline & & & 10.41 & 685002 & 0.92 & \\
\hline & & & 10.44 & 685004 & & \\
\hline & & & 10.59 & 140603308 & - & \\
\hline \multirow[t]{5}{*}{5} & \multirow[t]{5}{*}{ positive } & \multirow[t]{5}{*}{5} & 5.72 & 197121 & \multirow[t]{2}{*}{-} & \multirow{5}{*}{6.17} \\
\hline & & & 6.87 & 685001 & & \\
\hline & & & 6.61 & 685002 & \multirow[t]{3}{*}{2.25} & \\
\hline & & & 6.32 & 685004 & & \\
\hline & & & 6.45 & 140603308 & & \\
\hline 33 & positive & 25 & 23.36 & 685004 & - & - \\
\hline 34 & positive & 20 & 18.9 & 685004 & - & - \\
\hline
\end{tabular}




\section{Conclusion}

In conclusion, Raman is an ideal method for noninvasive screening of HSA injectable products. And the method was proved to be feasible and efficient for screening HSA injections, especially on its screening speed and the consideration of glass containers. Moreover, with inspiring results on the model transfer, the method could be used as a universal screening means to different Raman spectrometers. And the possibility of on-site screening of HSA products will be of great significance on the drug-safety regulation.

\section{Acknowledgments}

This research was supported by Youth Development Research Foundation (No. 2015C03) of National Institutes of Food and Drug Control, P. R. China. The findings and conclusion of this paper have not been formally disseminated by the National Institutes of Food and Drug Control (NIFDC) and should not be construed to represent Agency determination or policy.

\section{References}

1. L. Xiao, Q. Shen, "Establishment and evaluation of rapid identification method of human albumin preparations," China Pharmaceut. Affairs 21, 589-591 (2007).

2. Y. He, T. T. Ning, T. T. Xie, Q. C. Qiu, L. P. Zhang, Y. F. Sun, D. M. Jiang, K. Fu, F. Yin, W. J. Zhang, L. Shen, H. Wang, J. J. Li, Q. S. Lin, Y. X. Sun, H. Z. Li, Y. G. Zhu, D. C. Yang, "Large-scale production of functional human serum albumin from transgenic rice seeds," 108, 19078-19083 (2011).

3. D. D. Rutstein, E. F. Ingenito, W. E. Reynolds, J. M. Burke, "The determination of albumin in human blood plasma and serum. A method based on the ineraction of albumin with an anionic dye -2 (4'-hydroxybenzeneazo) benzoic acid," J. Clin. Invest. 33, 211-221 (1954).

4. E. Domenici, C. Bertucci, P. Salvadori, G. Félix, I. Cahagne, S. Motellier, I. W. Wainer, "Synthesis and chromatographic properties of an HPLC chiral stationary phase based upon human serum albumin," Chromatographia 29, 170-176 (1990).

5. M. Girard, A. Puerta, J. Carlos, M. D. Frutos, "High resolution separation methods for the determination of intact humanerythropoiesis stimulating agents. A review," Anal. Chim. Acta 713, 7-22 (2012).

6. M. Girard, H. P. Bietlot, N. Mousseau, T. D. Cyr, J.-C. Ethier, "Use of capillary electrophoresis for the characterization of human serum albumin heterogeneity," Biomed. Chromatogr. 12, 183-184 (1998).

7. Y. Wang, S. H. Wang, J. G. Chen, "Rapid identification of human albumin injections by Raman spectroscopy," Chin. J. Pharm. Anal. 32, 537-540 (2012).

8. D. Z. Mao, X. X. Wang, Y. J. Yang, "Determination of Human Albumin by Raman spectroscopy," Chin. J. Pharmaceut. 43, 776-779 (2012).

9. Y. Zhao, N. Ji, L. H. Yin, J. Wang, "A non-invasive method for the determination of liquid injectables by Raman spectroscopy," AAPS Pharm. Sci. Tech. 16, 914-921 (2015).

10. Y. Zhao, N. Ji, L. H. Yin, L. Zhu, X. B. Zhang, "Establishment of a Raman database for non-invasive and rapid screening of liquid injectables," Chin. J. Pharm. Anal. 35, 1263-1273 (2015).

11. L. H. Yin, Y. Zhao, N. Ji, J. Wang, Y. J. Gao, X. B. Zhang, L. Zhu, "A method for determination of liquid injectables by Raman spectroscopy," Patent of invention, ZL201210593760.9, China (2015).

12. Y. Zhao, L. H. Yin, N. Ji, Y. J. Gao, J. Wang, L. Zhu, X. B. Zhang, "A method for eliminating the differences between Raman spectrometers," Patent of invention, ZL201310048251.2, China (2015). 